\title{
Genome-wide association study between chickpea accessions from VIR collection and phenotypic data
}

\author{
A.B. Sokolkova ${ }^{1 *}$, M.A. Vishnyakova ${ }^{2}$, S. Bulyntsev' ${ }^{2}$, P.L. Chang ${ }^{3,4}$, \\ N. Carrasquilla-Garcia ${ }^{4}$, M.G. Samsonova ${ }^{1}$, S.V. Nuzhdin ${ }^{1,3}$ \\ ${ }^{1}$ Peter the Great St. Petersburg Polytechnic University, St. Petersburg, Russia \\ ${ }^{2}$ Federal Research Centre All-Russian N.I. Vavilov Institute of Plant Genetic Resources (VIR), \\ St. Petersburg, Russia \\ ${ }^{3}$ University of Southern California, Los Angeles, CA, USA \\ ${ }^{4}$ Department of Plant Pathology, University of California, Davis, CA, USA \\ *e-mail:alyonasok@yandex.ru
}

Key words: chickpea (Cicer arietinum L.), GWAS analysis, candidate genes

The Vavilov Institute of Plant Genetic Resources (VIR), in St. Petersburg, Russia, houses a unique genebank of chickpea (Cicer arietinum L.). Genotyping by sequencing of VIR's 428 chickpea accessions from different countries identified 56,855 segregating single nucleotide polymorphisms (SNP). For these SNP calls we implemented inclusion criteria: minor allele frequency (MAF) more than $3 \%$, genotype call-rate more than $90 \%$. In addition, we performed imputation of missing values. We performed Genome-wide association study (GWAS) to find associations (for data with and without imputation) between VIR's chickpea accessions and phenotypic data obtained in Kuban experimental station of VIR in 2016 and 2017. GWAS analysis identified a large number of genome intervals and potential gene candidates that may affect important agronomic traits. Besides that, we made a link between traits measured in GWAS and specific climatic variables at collection sites. 